\title{
Review of: "Restricted cement augmentation in unstable geriatric midthoracic fractures treated by long-segmental posterior stabilization leads to a comparable construct stability"
}

Sheng Yang

Potential competing interests: The author(s) declared that no potential competing interests exist.

This is an interesting study. Ulrich J Spiegel had another similar study in 2020(Spiegl UJ, Weidling M, Schleifenbaum S, Reinhardt M, Heyde CE. Comparison of Long Segmental Dorsal Stabilization with Complete Versus Restricted Pedicle Screw Cement Augmentation in Unstable Osteoporotic Midthoracic Vertebral Body Fractures: A Biomechanical Study. World Neurosurg. 2020 Nov;143:e541-e549. doi: 10.1016/j.wneu.2020.08.002. Epub 2020 Aug 8. PMID: 32777399).

The results showed that ResPSCA had similar stability to ComPSCA in the treatment of unstable osteoporotic mid-thoracic fractures with long-segment pedicle screw fixation. And the results remind orthopaedic surgeons to re-examine the core and essence of bone-cement enhancement. Bone cement reinforcement not only increases the fixation strength of screws, but also limits the range of motion of spine. ResPSCA provides stability similar to ComPSCA by limiting the movement of the proximal and distal ends of the fixation segment and reducing the increased screw stress due to forward bending. This is similar to our view that using a thoracolumbar orthosis (TLSO) to limit spinal movement after shortsegment pedicle screw internal fixation for unstable thoracolumbar fractures can help avoid fixation failure.

The risk factors of pedicle screw fixation failure in unstable thoracolumbar fractures are mainly related to the degree of primary fracture injury and vertebral bone abnormality (such as osteoporosis/diffuse idiopathic skeletal hypertrophy). Lengthening fixation segment and bone cement reinforcement are commonly used methods to reduce fixation failure. Whether the range of motion (especially flexion) of the spine is a risk factor for the failure of pedicle screw fixation for unstable fractures has not been concerned. Our series of clinical and biomechanical studies (finite element analysis) of thoracolumbar fractures suggest that flexion is one of the risk factors for failure of short-segment pedicle screw fixation in unstable type A fractures.

The results of this study are mutually verified with our research to a certain extent. A quantifiable and reproducible fracture model closer to the clinical classification of thoracolumbar fractures is the basis for 
further research. In addition, the comparative analysis of pedicle screw stress and injured vertebral displacement under vertical and anterior flexion conditions of thoracolumbar fractures is also necessary. 